\title{
Intravascular Large B-cell Lymphoma of the Bilateral Ovaries and Uterus in an Asymptomatic Patient with a $t(11 ; 22)(q 23 ; q 11)$ Constitutional Translocation
}

\author{
Yasuyuki Shigematsu ${ }^{1,2}$, Motoki Matsuura ${ }^{3}$, Noriko Nishimura ${ }^{1}$, Naoko Tsuyama ${ }^{4}$, \\ Kengo Takeuchi ${ }^{4,5}$, Yasuhito Terui ${ }^{1}$, Nobuhiro Takeshima ${ }^{3}$ and Kiyohiko Hatake ${ }^{1}$
}

\begin{abstract}
Intravascular large B-cell lymphoma (IVLBCL) is a rare subtype of extranodal diffuse large B-cell lymphoma, which is characterized by the intravascular growth of lymphoma cells, aggressive behavior, and an often fatal course. Patients with IVLBCL are usually symptomatic. Although any organ may be involved, localized lesions in the bilateral ovaries and uterus are extremely rare. We experienced a rare case of IVLBCL involving the bilateral ovaries and uterus in an asymptomatic patient with a $\mathrm{t}(11 ; 22)(\mathrm{q} 23 ; \mathrm{q} 11)$ constitutional balanced translocation. Its association with the disease remains unknown. Even in asymptomatic situations, IVLBCL is possible, and the uterus and ovaries can be involved.
\end{abstract}

Key words: IVLBCL, bilateral ovaries, uterus, $t(11 ; 22)$ constitutional translocation

(Intern Med 55: 3169-3174, 2016)

(DOI: 10.2169/internalmedicine.55.6578)

\section{Introduction}

Intravascular large B-cell lymphoma (IVLBCL) is a rare aggressive subtype of B-cell lymphoma which is characterized by the proliferation of malignant lymphocytes within the lumen of small blood vessels and without an extravascular tumor mass (1). The clinical presentation shows tremendous variation: anemia, neurological symptoms, hepatomegaly, and splenomegaly; and constitutional B symptoms (fever, night sweats, and weight loss) are observed in the majority of patients (2-4). Thus, IVLBCL is usually symptomatic. The aggressive clinical behavior of the disease often results in a delayed diagnosis and fatal complications due to the occlusion of blood vessels (5). The prognosis is poor, with the 3-year survival rate reported to be between 14 and $27 \%(3,6)$. Although any organ can be involved, localized lesions in the bilateral ovaries and uterus are extremely rare (7-11).
The $\mathrm{t}(11 ; 22)(\mathrm{q} 23 ; \mathrm{q} 11)$ translocation, which is the most common recurrent non-Robertsonian constitutional balanced translocation in humans, has been reported in more than 160 unrelated families (12). Carriers of the $\mathrm{t}(11 ; 22)(\mathrm{q} 23 ; \mathrm{q} 11)$ constitutional translocation manifest no clinical symptoms; however, they often have problems with reproduction (13). The $\mathrm{t}(11 ; 22)(\mathrm{q} 23 ; \mathrm{q} 11)$ constitutional translocation has also been reported to be associated with an increased risk of breast cancer $(14,15)$, while the association between the translocation and IVLBCL remains unknown.

We experienced a case of IVLBCL involving the bilateral ovaries and uterus in an asymptomatic patient with a $\mathrm{t}(11 ; 22)(\mathrm{q} 23 ; \mathrm{q} 11)$ constitutional translocation. To the best of our knowledge, there have been no previous reports of such a case. We present this case with a discussion of the diagnosis and the treatment strategies.

\footnotetext{
${ }^{1}$ Division of Hematology Oncology, The Cancer Institute Hospital, Japanese Foundation of Cancer Research, Japan, ${ }^{2}$ Division of General Oncology, The Cancer Institute Hospital, Japanese Foundation of Cancer Research, Japan, ${ }^{3}$ Division of Gynecologic Oncology, The Cancer Institute Hospital, Japanese Foundation of Cancer Research, Japan, ${ }^{4}$ Division of Pathology, The Cancer Institute Hospital, Japanese Foundation of Cancer Research, Japan and ${ }^{5}$ Pathology Project for Molecular Targets, The Cancer Institute, Japanese Foundation for Cancer Research, Japan Received for publication September 16, 2015; Accepted for publication December 9, 2015 Correspondence to Dr. Kiyohiko Hatake, khatake@jfcr.or.jp
} 


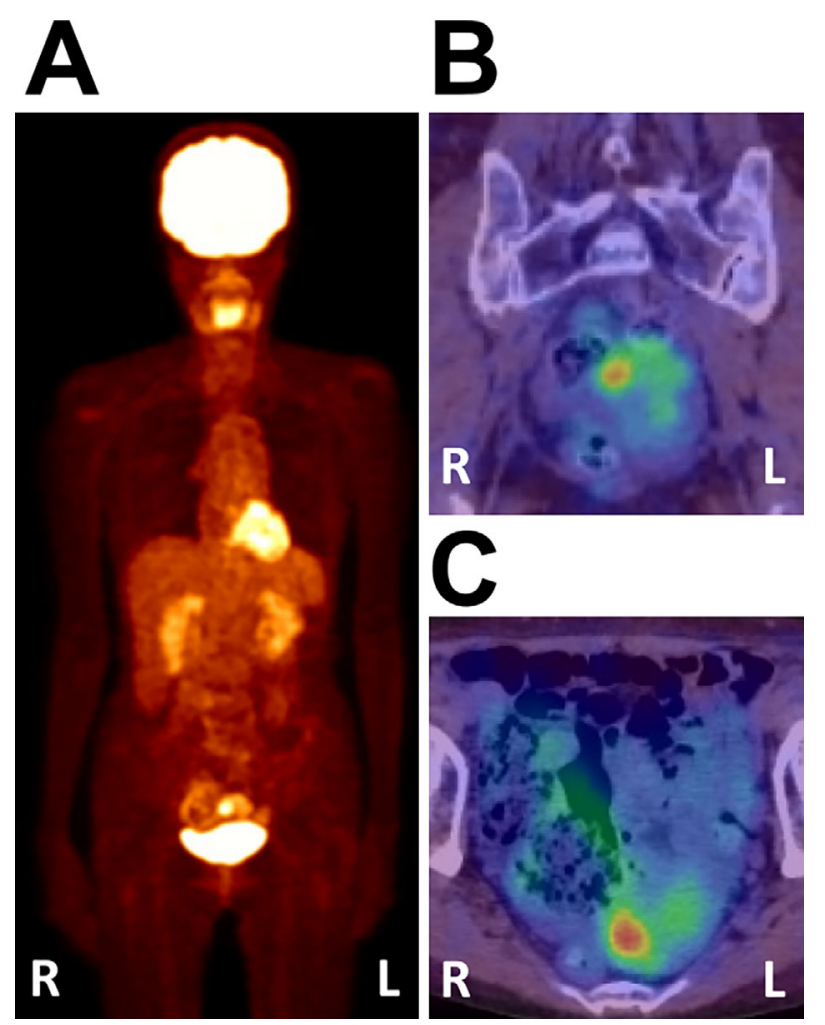

Figure 1. Fluorodeoxyglucose (FDG) -positron emission tomography (PET) and PET/computed tomography (CT) fusion images. (A) A PET image showing increased FDG uptake only in the intrapelvic region. (B) Coronal and (C) axial views of PET/CT reveal the accumulation of FDG in the fundus of the uterus. L: left, R: right

\section{Case Report}

A 72-year-old Japanese female without any symptoms underwent an annual positron emission tomography (PET)computed tomography (CT) examination for opportunistic cancer screening, and metabolically active foci with maximal standardized uptake values of six were found in the fundus and front wall of the uterus (Fig. 1). Contrast-enhanced magnetic resonance imaging (MRI) was conducted as a more thorough examination. Although it revealed marked early enhancement in the uterus, few endometrial abnormalities were observed (Fig. 2). The imaging studies showed no abnormalities in the bilateral ovaries, abdominal lymph nodes or peritoneal membrane. In addition, the patient's epithelial tumor marker levels - including the carcinoembryonic antigen, carbohydrate antigen 19-9, carbohydrate antigen 125 , and squamous cell carcinoma antigen levels - were all within normal limits. These findings suggested that uterine sarcoma or malignant lymphoma were more likely diagnoses than endometrial carcinoma. The patient was referred to our hospital for a workup and treatment with a provisional diagnosis of uterine sarcoma, malignant lymphoma, and atypical endometrial carcinoma. Although her past medical history revealed no significant medical illness, she had experienced an early fetal death and a miscarriage at 29 and 33 years of age, respectively.

A pelvic examination and transvaginal ultrasound at our hospital revealed no abnormalities. To eliminate the possibility of a uterine metastasis from an intestinal malignancy, upper and lower gastrointestinal endoscopies were performed. These revealed no significant findings. The only abnormality in the patient's laboratory data was a significant increase in her lactic dehydrogenase (LDH; 519 U/L) level. A cytological examination of the cervix and uterine mucosa revealed the presence of non-epithelial malignant cells (Fig. 3). The cytological features, imaging findings, and her clinical presentation suggested that uterine sarcoma was more likely than malignant lymphoma. A total abdominal hysterectomy with bilateral salpingo-oophorectomy was performed as a diagnostic therapy. The pathological findings showed that the lymphoma cells were almost exclusively contained within the blood vessels, including the capillaries, without an obvious extravascular tumor mass (Fig. 4). A diagnosis of IVLBCL involving the bilateral ovaries and uterus was made. The lymphoma cells were positive for CD20, CD5, BCL2, BCL6, and MUM1, and negative for CD10, CyclinD1, and SOX11, suggesting a non-germinal center B-cell origin. Although IVLBCL is likely to involve the central nervous system (CNS), the results of contrast brain MRI and a cerebrospinal fluid analysis did not demonstrate CNS involvement. A bone marrow analysis revealed hypocellular marrow with no lymphoma cells, and a Giemsa-band analysis showed 46,XX,t(11;23)(q23;q11.2) translocation in all of the analyzed cells, suggesting that the translocation was constitutional.

We planned to administer R-CHOP, a combination chemotherapy consisting of cyclophosphamide, doxorubicin, vincristine, and prednisolone with rituximab (a recombinant anti-CD20 antibody) once every three weeks for six cycles. Although the patient had few findings that would indicate CNS involvement, we also planned to administer intrathecal prophylaxis (methotrexate, cytarabine, and prednisolone) once every three weeks for four cycles. After one cycle of chemotherapy, the soluble interleukin-2 receptor (sIL-2R) and LDH levels decreased to $1,076 \mathrm{U} / \mathrm{mL}$ and $167 \mathrm{U} / \mathrm{L}$, respectively. The patient has been treated on an outpatient basis and has maintained a good performance status with the chemotherapy. Thus far, no disease progression has been recognized.

\section{Discussion}

The present case shows that IVLBCL involving the bilateral ovaries and uterus was incidentally found in an asymptomatic patient with a $\mathrm{t}(11 ; 22)(\mathrm{q} 23 ; \mathrm{q} 11)$ constitutional translocation. The lack of lymph node swellings and hepatosplenomegaly without B symptoms made a diagnosis of malignant lymphoma seem less likely. The localized FDG accumulation in the fundus of the uterus, the presence of few morphological changes in the endometrium and the cy- 

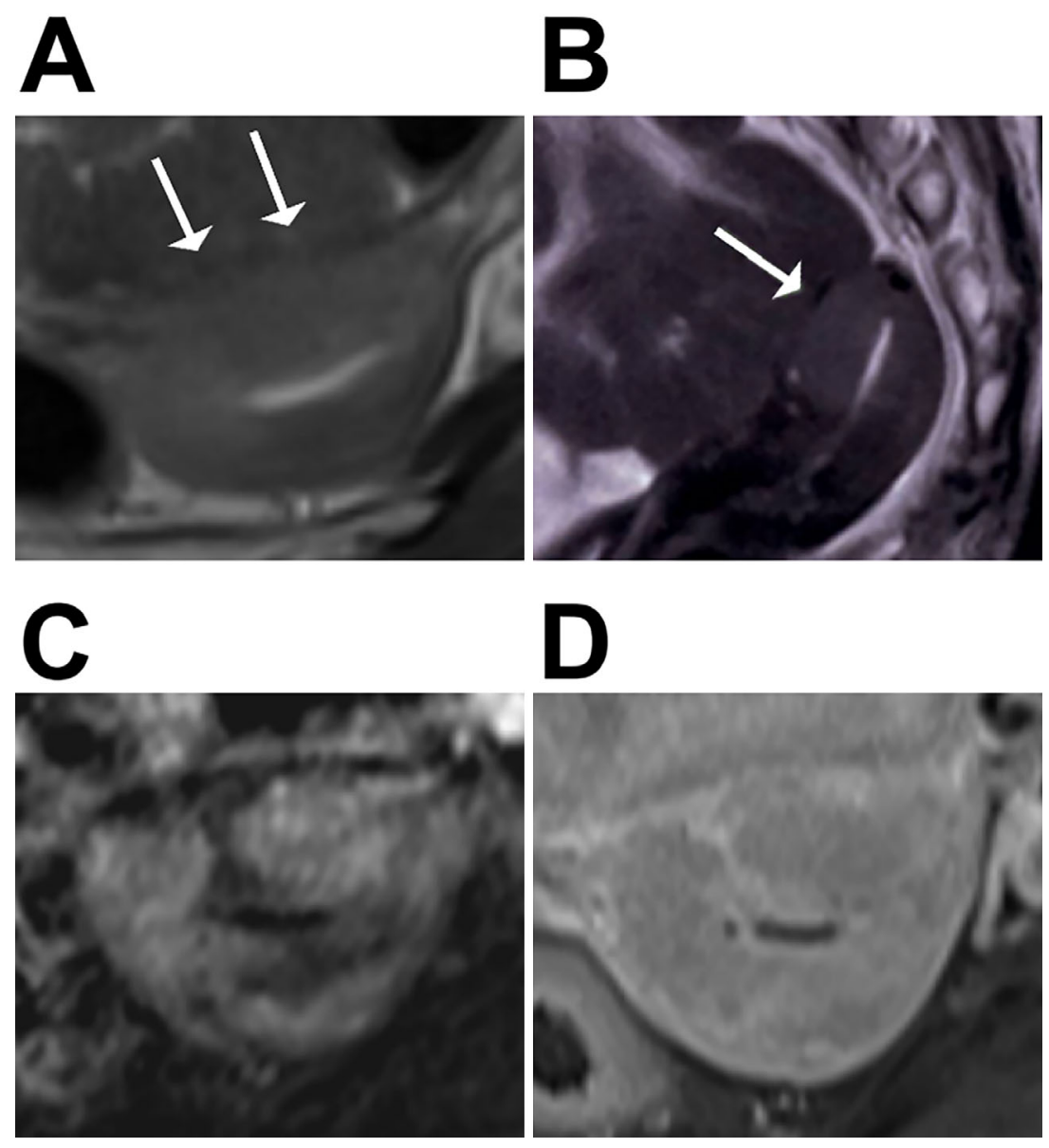

Figure 2. T2-weighted fast spin-echo and dynamic gadolinium-enhanced magnetic resonance imaging (MRI). (A) Axial and (B) sagittal views of T2-weighted fast spin-echo MRI show few abnormalities in the uterine endometrium. There are few findings to indicate the presence of extra-uterine invasion (arrows). A dynamic MRI scan showing (C) early enhancing lesions in the fundic myometrium. (D) In the late phase, the lesions show relatively lower intensity than in the other area of the myometrium. These findings suggest that uterine sarcoma or malignant lymphoma are more likely than endometrial carcinoma.

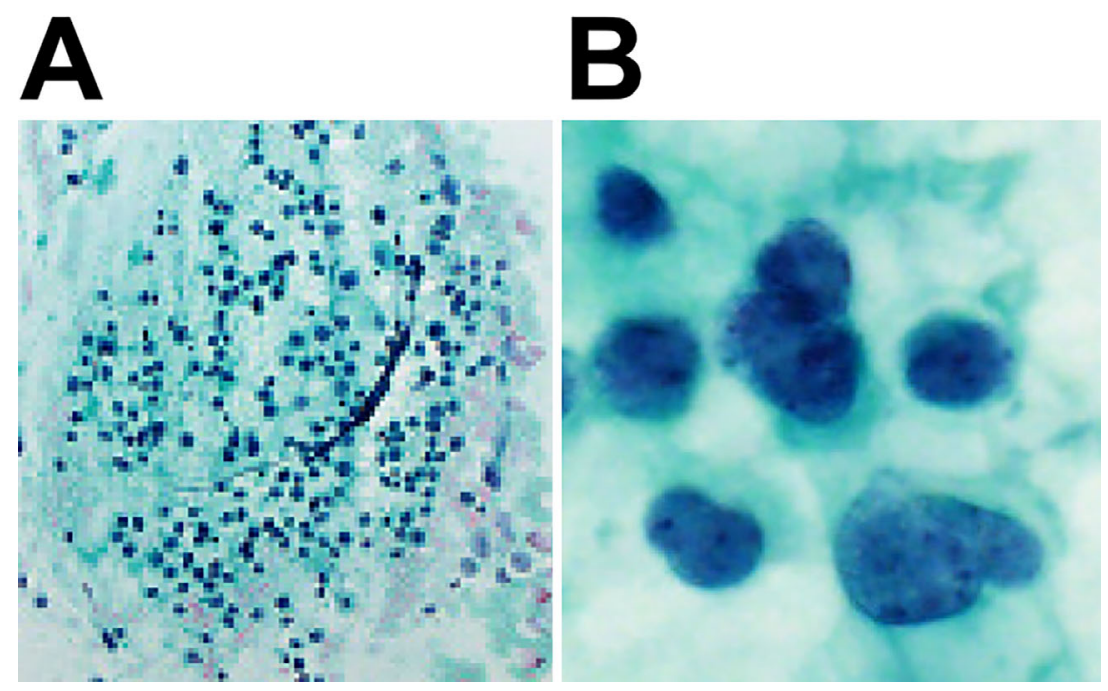

Figure 3. A cytological analysis of the endometrium. (A) Papanicolaou staining shows that the cells were arranged in a predominantly single cell pattern with naked nuclei $(\times 100)$. (B) The cells with cytoplasm demonstrated high nuclear to cytoplasmic ratios. Their morphology varied, and nuclear pleomorphisms, such as binucleated forms could be seen. These findings suggest that the cells were clearly malignant and that a diagnosis of endometrial carcinoma was less likely $(\times 400)$. 
A

B
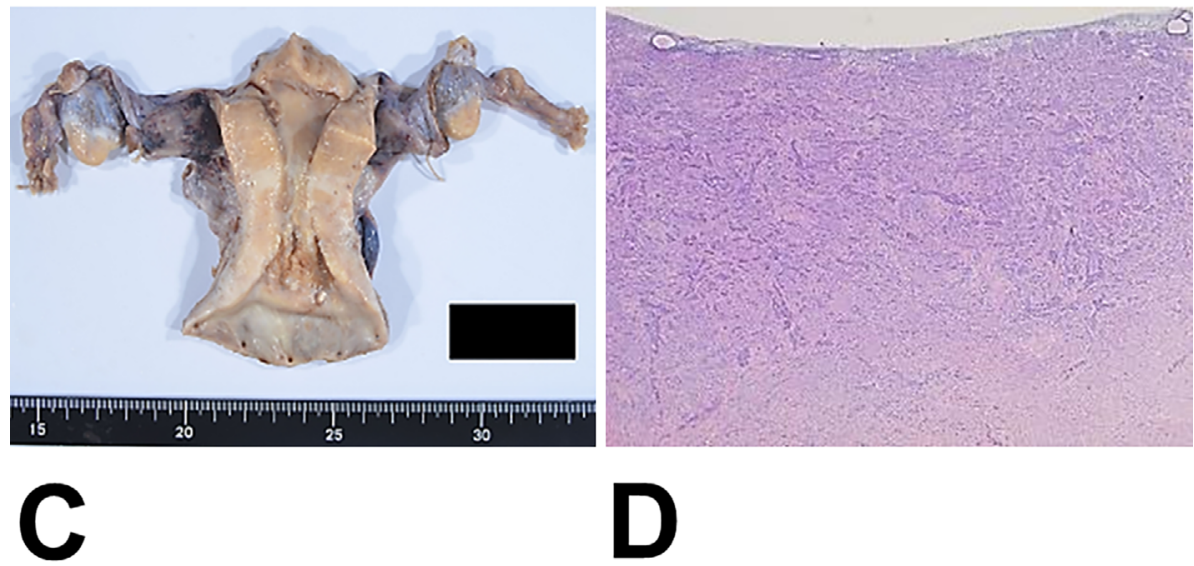

D
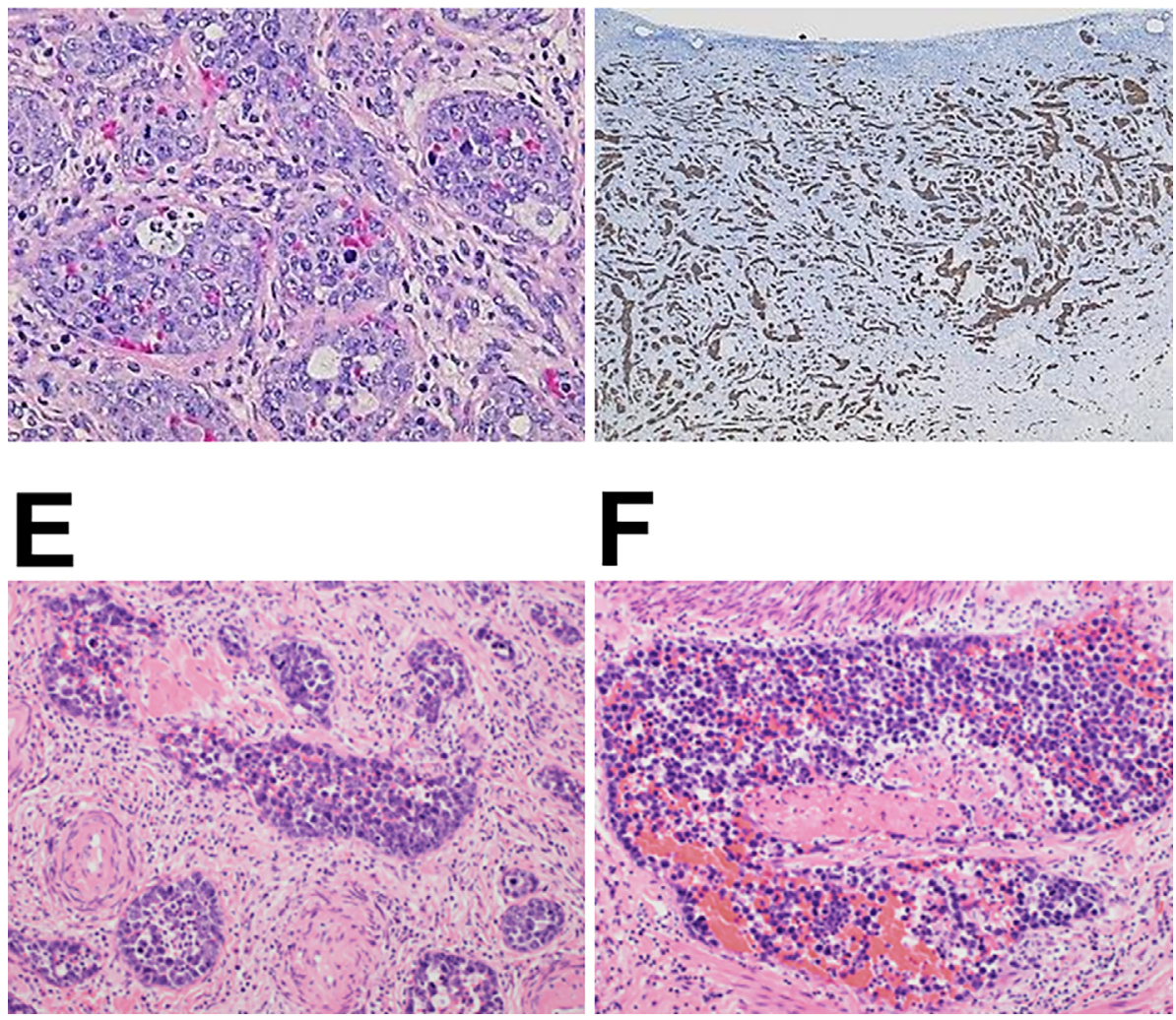

Figure 4. Macroscopic and histopathological examinations. (A) A macroscopic view of the uterus and the ovaries revealed their normal appearance without any evidence of tumor. (B) Hematoxylin and Eosin staining sections of the uterus $(\times 20)$ and $(C)$ a higher magnification image $(\times 400)$ shows that the blood vessels were filled with lymphoma cells. (D) The tumor cells in the uterine vessels were highlighted by immunohistochemical staining for CD20 $(\times 20)$. Capillaries in the right $(E)$ and left $(F)$ ovaries were also filled with lymphoma cells $(\times 200)$.

tological findings suggested that uterine sarcoma was more likely than endometrial carcinoma. Only the surgical specimens of the bilateral ovaries and uterus revealed the presence of IVLBCL, which had spread throughout the uterine and ovarian blood vessels. To the best of our knowledge, this is the first report of IVLBCL involving the bilateral ovaries and uterus to be diagnosed in an asymptomatic patient.

Interestingly, the clinical presentation of IVLBCL appears to differ according to the country of origin. Two main clini- cal variants are recognized: the Western and Asian variants. In the Western variant, the CNS (34-100\%) and skin (39$60 \%$ ) are most commonly involved $(4,6,16)$; on the other hand, the myeloid and lymphoid system, such as the bone marrow (32\%) and spleen (29\%) are less frequently involved (4). In contrast, in the Asian variant, the involvement of the bone marrow $(75 \%)$, spleen $(67 \%)$, and liver $(55 \%)$ is often observed $(3,17,18)$, while neurological symptoms $(27 \%)$ and skin lesions (15\%) are less common (19). However, in our patient, a physical examination, brain contrast 
MRI, bone marrow biopsy, lumbar puncture, and an abdominal enhanced CT scan, yielded no relevant findings.

It is impossible to diagnose IVLBCL on the basis of cytological findings $(8,20-22)$. In this case, the presence of features such as dissociated single cells with high nuclear and cytoplasmic ratios, as well as nuclear pleomorphisms with prominent nucleoli, suggested the presence of non-epithelial malignant cells. In such cells, the cytological features associated with some types of sarcoma are quite similar to those of malignant lymphoma. Thus, cytological findings are sometimes insufficient for distinguishing between the two conditions. When cytological findings that suggest the presence of non-epithelial malignant cells are obtained, the possibility of malignant lymphoma should be considered. A histological analysis is essential for the final diagnosis.

Abnormal laboratory findings, such as increases in $\mathrm{LDH}$ and sIL-2R, anemia, and an elevated sedimentation rate have been reported in more than half of IVLBCL patients. However, these findings are not specific for IVLBCL (1). Our patient also presented markedly elevated LDH and sIL-2R levels at the initial visit; these abnormally high levels continued, even after the surgery in which the metabolically active foci which were found on PET-CT were completely resected. Although PET-CT has been reported to be useful in the diagnosis of IVLBCL, false-negative results for some types of organ involvement, such as skin and renal involvement, were also recognized. The exact sensitivity of PET-CT for the detection of lymphoma cells in IVLBCL remains unknown because of the rarity of the condition, and careful interpretation is required.

IVLBCL is primarily treated with systemic chemotherapy with rituximab and CNS-directed therapy. Although there are no standard chemotherapy regimens for IVLBCL, anthracycline-containing regimens have been reported to improve the clinical outcome (6). The largest retrospective analysis of treatment, in which approximately $83 \%$ of the patients received $\mathrm{CHOP}$ or $\mathrm{CHOP}-$ like chemotherapy, with or without rituximab (2), demonstrated that the patients who received chemotherapy plus rituximab had a significantly higher complete response ( $82 \%$ versus $51 \%$ ) and two-year overall survival $(66 \%$ versus $46 \%)$ rate than patients who did not receive rituximab, indicating the efficacy of rituximab. As for therapies directed at the CNS, we administered CNS-directed therapy as a prophylactic treatment against potential CNS metastasis in the present case (despite the patient showing few signs of CNS involvement) because IVLBCL is associated with a high CNS relapse rate [the incidence during follow-up is reported to be $25 \%$ (19)].

The $\mathrm{t}(11 ; 22)(\mathrm{q} 23 ; \mathrm{q} 11.2)$ is the most frequent constitutional balanced translocation in humans (12). Few reports have shown an association between the translocation and IVLBCL; on the other hand, the translocation appears to have a significant association with breast cancer $(14,15)$. To the best of our knowledge, this is the first report of an IVLBCL patient with this translocation.

In conclusion, we experienced a rare case of IVLBCL in- volving the bilateral ovaries and uterus in an asymptomatic patient with a $\mathrm{t}(11 ; 22)$ constitutional translocation. The present case demonstrates that IVLBCL may occur in asymptomatic patients and that the uterus and the ovaries can be involved. The diagnosis of IVLBCL is challenging because the laboratory and imaging studies show low specificity, and the cytological findings are limited. There is room for considering the possibility of IVLBCL when unexplained clinical findings are observed in patients with elevated LDH and sIL-2R levels.

Written informed consent was obtained from the patient for publication of this case report and any accompanying images and laboratory data.

\section{The authors state that they have no Conflict of Interest (COI).}

\section{Acknowledgement}

The authors thank Dr. Masahiro Yokoyama, Dr. Yuko Mishima, Dr. Kyoko Ueda, and Dr. Hideaki Nitta for their helpful discussions and the critical reading of the manuscript, and Mr. Koichi Ikehata for the preparation of cytology specimens and his constructive comments regarding the figure legend.

\section{References}

1. Ponzoni M, Ferreri AJM, Campo E, et al. Definition, diagnosis, and management of intravascular large B-cell lymphoma: proposals and perspectives from an international consensus meeting. J Clin Oncol 25: 3168-3173, 2007.

2. Shimada K, Matsue K, Yamamoto K, et al. Retrospective analysis of intravascular large B-cell lymphoma treated with rituximabcontaining chemotherapy as reported by the IVL study group in Japan. J Clin Oncol 26: 3189-3195, 2008.

3. Murase T, Yamaguchi M, Suzuki R, et al. Intravascular large Bcell lymphoma (IVLBCL): a clinicopathologic study of 96 cases with special reference to the immunophenotypic heterogeneity of CD5. Blood 109: 478-485, 2007.

4. Ferreri AJM, Campo E, Seymour JF, et al. Intravascular lymphoma: clinical presentation, natural history, management and prognostic factors in a series of 38 cases, with special emphasis on the "cutaneous variant". Br J Haematol 127: 173-183, 2004.

5. Shimada K, Kinoshita T, Naoe T, Nakamura S. Presentation and management of intravascular large B-cell lymphoma. Lancet Oncol 10: 895-902, 2009.

6. DiGiuseppe JA, Nelson WG, Seifter EJ, Boitnott JK, Mann RB. Intravascular lymphomatosis: a clinicopathologic study of 10 cases and assessment of response to chemotherapy. J Clin Oncol 12: 2573-2579, 1994.

7. Xia Y, Wang Y, Jiang Y, et al. Primary intravascular large B cell lymphoma of the endometrium. Acta Histochem 116: 993-996, 2014.

8. Yamamoto $\mathrm{T}$, Morita $\mathrm{K}$, Iriyama $\mathrm{N}$, et al. Intravascular large B-cell lymphoma of the uterus: a case with favorable clinical outcome. Int J Surg Pathol 19: 672-676, 2011.

9. Sur M, Ross C, Moens F, Daya D. Intravascular large B-cell lymphoma of the uterus: a diagnostic challenge. Int J Gynecol Pathol 24: 201-203, 2005.

10. Yamada N, Uchida R, Fuchida S, et al. $\mathrm{CD}^{+}$Epstein-Barr viruspositive intravascular large B-cell lymphoma in the uterus coexisting with huge myoma. Am J Hematol 78: 221-224, 2005.

11. Lannoo L, Smets S, Steenkiste E, et al. Intravascular large B-cell 
lymphoma of the uterus presenting as fever of unknown origin (FUO) and revealed by FDG-PET. Acta Clin Belg 62: 187-190, 2007.

12. Fraccaro M, Lindsten J, Ford CE, Iselius L. The 11q;22q translocation: a European collaborative analysis of 43 cases. Hum Genet 56: $21-51,1980$.

13. Sugiura-Ogasawara M, Ozaki Y, Sato T, Suzumori N, Suzumori K. Poor prognosis of recurrent aborters with either maternal or paternal reciprocal translocations. Fertil Steril 81: 367-373, 2004.

14. Lindblom A, Sandelin K, Iselius L, et al. Predisposition for breast cancer in carriers of constitutional translocation 11q;22q. Am J Hum Genet 54: 871-876, 1994.

15. Wieland I, Muschke P, Volleth M, et al. High incidence of familial breast cancer segregates with constitutional $t(11 ; 22)(\mathrm{q} 23 ; \mathrm{q} 11)$. Genes Chromosomes Cancer 45: 945-949, 2006.

16. Calamia KT, Miller A, Shuster EA, Perniciaro C, Menke DM. Intravascular lymphomatosis. A report of ten patients with central nervous system involvement and a review of the disease process. Adv Exp Med Biol 455: 249-265, 1999.

17. Narimatsu H, Morishita $Y$, Saito $S$, et al. Usefulness of bone marrow aspiration for definite diagnosis of Asian variant of intravascular lymphoma: four autopsied cases. Leuk Lymphoma 45: 16111616, 2004.

18. Murase T, Nakamura S, Kawauchi K, et al. An Asian variant of intravascular large B-cell lymphoma: clinical, pathological and cytogenetic approaches to diffuse large B-cell lymphoma associated with haemophagocytic syndrome. Br J Haematol 111: 826-834, 2000.

19. Shimada K, Murase T, Matsue $K$, et al. Central nervous system involvement in intravascular large B-cell lymphoma: a retrospective analysis of 109 patients. Cancer Sci 101: 1480-1486, 2010.

20. Fujiwara A, Nagayama S, Amada S, Shimamoto T, Shimao Y, Hayashi T. Intravascular large B-cell lymphoma involving mainly the uterus: report of a case using liquid-based cytology of the endometrium. Acta Cytol 54: 787-792, 2010.

21. Nakamichi I, Shimazu K, Ikeda J, et al. Intravascular lymphomatosis initially suspected from uterine cytology: a case report. Acta Cytol 53: 198-200, 2009.

22. Ohwada M, Suzuki M, Onagawa T, Ishida A, Kawai T, Sato I. Primary malignant lymphoma of the uterine corpus diagnosed by endometrial cytology. A case report. Acta Cytol 44: 1045-1049, 2000.

The Internal Medicine is an Open Access article distributed under the Creative Commons Attribution-NonCommercial-NoDerivatives 4.0 International License. To view the details of this license, please visit (https://creativecommons.org/licenses/ by-nc-nd/4.0/).

(C) 2016 The Japanese Society of Internal Medicine http://www.naika.or.jp/imonline/index.html 